\title{
ANN Based Support for Distributed Energy Resources Scheduling in Smart Grids
}

\author{
Zita Vale* Hugo Morais* \\ Pedro Faria* \\ * GECAD - Knowledge Engineering and Decision-Support Research Group \\ Institute of Engineering - Polytechnic of Porto (ISEP/IPP) \\ Rua Dr. António Bernardino de Almeida, 431, 4200-072 Porto \\ Portugal (Tel: +351-228340500; e-mail: \{zav, hgvm,pnf $\} @$ isep.ipp.pt).
}

\begin{abstract}
The future scenarios for operation of smart grids are likely to include a large diversity of players, of different types and sizes. With control and decision making being decentralized over the network, intelligence should also be decentralized so that every player is able to play in the market environment. In the new context, aggregator players, enabling medium, small, and even micro size players to act in a competitive environment, will be very relevant.

Virtual Power Players (VPP) and single players must optimize their energy resource management in order to accomplish their goals. This is relatively easy to larger players, with financial means to have access to adequate decision support tools, to support decision making concerning their optimal resource schedule. However, the smaller players have difficulties in accessing this kind of tools. So, it is required that these smaller players can be offered alternative methods to support their decisions.

This paper presents a methodology, based on Artificial Neural Networks (ANN), intended to support smaller players' resource scheduling. The used methodology uses a training set that is built using the energy resource scheduling solutions obtained with a reference optimization methodology, a mixed-integer non-linear programming (MINLP) in this case. The trained network is able to achieve good schedule results requiring modest computational means.
\end{abstract}

\begin{tabular}{|c|c|c|c|}
\hline & NOMENCLATURE & $P_{\text {LoadCut }}$ & Load curtailment power \\
\hline$c_{\text {ExcessGeneratedEnergy }}$ & Excess generated energy cost & $P_{\text {LoadCutLimit }}$ & Load curtailment power limit \\
\hline$c_{\text {LoadCut }}$ & Load curtailment cost & $P_{\text {LoadRed }}$ & Load reduction power \\
\hline$c_{\text {LoadRed }}$ & Load reduction cost & $P_{\text {LoadRedLimit }}$ & Load reduction power limit \\
\hline$c_{\text {Main }}$ & Main network energy cost & $P_{\text {Main }}$ & Main network power \\
\hline$c_{\text {Other }}$ & Other generation cost & $P_{\text {Other }}$ & Other generation power \\
\hline$c_{\text {Photovoltaic }}$ & Photovoltaic generation cost & $P_{\text {OtherLimit }}$ & Other generation power limit \\
\hline$c_{\text {StorageCharge }}$ & Storage charge cost & $P_{\text {Photovoltaic }}$ & Photovoltaic power \\
\hline$c_{\text {StorageDischarge }}$ & Storage discharge cost & $P_{\text {PhotovoltaicLimit }}$ & Photovoltaic power limit \\
\hline$c_{\text {UndeliveredEnergy }}$ & Undelivered energy cost & $P_{\text {Storage }}$ & Storage power \\
\hline$c_{\text {Wind }}$ & Wind generation cost & $P_{\text {Storagelnitial }}$ & Initial stored power \\
\hline$R_{r}^{\text {proposed }}$ & Result $R$ obtained with the proposed & $P_{\text {StorageCharge }}$ & Storage charge power \\
\hline & methodology for the result element $r$ & $P_{\text {StorageChargeLimit }}$ & Storage charge power limit \\
\hline$R_{r}^{\text {reference }}$ & $\begin{array}{l}\text { Result } R \text { obtained with the reference } \\
\text { methodology for the result element } r\end{array}$ & $P_{\text {StorageDischarge }}$ & Storage discharge power \\
\hline$E_{\text {xcessGeneratedEnergy }}$ & Excess generated energy & $P_{\text {StorageDischargeLimit }}$ & Storage discharge power limit \\
\hline$n r$ & Number of result elements & $P_{\text {StorageLimit }}$ & Storage power limit \\
\hline & Load nower & $P_{\text {Wind }}$ & Wind power \\
\hline & & $P_{\text {WindLimit }}$ & Wind power limit \\
\hline
\end{tabular}




$\begin{array}{ll}r & \text { Each element of results } \\ U_{\text {ndeliveredEnergy }} & \text { Undelivered energy power } \\ X_{\text {Storage }} & \text { Binary variable for storage charge } \\ X_{\text {Cut }} & \text { Binary variable for load curtailment } \\ Y_{\text {Storage }} & \text { Binary variable for storage discharge }\end{array}$

\section{INTRODUCTION}

Decision making concerning Distributed Energy Resources (DER) scheduling is of crucial importance in the context of smartgrids. DER use has gained greater and greater importance, namely in what concerns the increasing electrical energy generation from renewable energy primary sources. Increasing use of Renewable Energy Sources (RES) aims at reducing the impact of electrical energy generation, namely in what concerns Green House Gas (GHG) emissions.

In the first phase, the increasing use of RES has been almost entirely focussed on the implementation of RES based generation equipment. Although some RES based generation sites are large dimension plants (e.g. large hydroelectric), the total amount of RES based electrical energy generation is largely achieved due to a large number of micro, small and medium size plants.

Presently, some areas of the globe already achieved intensive levels of DER penetration. In 2009 RES represented $25 \%$ of the global electrical power capacity, $18 \%$ of power production, $60 \%$ of newly installed capacity in Europe, and more than $50 \%$ of newly installed capacity in USA (REN21, 2010). At this point, it is certainly time to focus on the management of these resources. Adequate management of DER is of most importance for Power Systems (PS) efficiency and is especially relevant in the case of smartgrids as a diversity of resources of different characteristics and nature, as well as diversity of size, are involved.

RES are based on natural resources, as wind, sun, and water, which have an intrinsic intermittent non-controllable nature (Milošević, 2005). With the intensive use of this kind of sources, the mutual adaptation between energy supply and energy consumption can be achieved using
a) energy storage
b) demand management.

Although a) and b) have been attracting attention since a long time ago, there is a significant technologic gap between the present state of the art and their practical use in a real world context. The importance of both a) and b) is clearly demonstrated by the present need of wind curtailment (Sioshansi et al., 2009)

This paper presents an insight on the issue of DER scheduling in the context of smartgrids. It departs from the optimal scheduling of DER, considering generation with an intensive of RES, storage and demand response (Morais, 2008; Vale, 2009; Vale, 2010).

Based on the optimal scheduling results already presented in previous papers (Morais, 2010), this paper analyses the most important relationships that can be established between known and forecasted data and the cost of the smartgrid operation.

Having in mind that a significant number of smartgrid players will be of small and medium size, it is important to note that these players will not have the complex computational means to directly use the optimal scheduling methodologies proposed in (Morais, 2010). In order to overcome this limitation, this paper proposes an ANN based methodology to support these small and medium size players' decisions.

Section 2 presents the proposed methodology for aggregators' decision-support. Section 3 presents a casestudy that analyses the results of operation costs and Locational Marginal Prices (LMP) in function of load and price variation. Finally, section 4 presents the most important conclusions of the paper.

\section{ANN-BASED PROPOSED METHODOLOGY}

This paper proposes a methodology for supporting smartgrid players' philosophy for the scheduling of their energy resources. In previous works, the authors have proposed optimal DER scheduling methodologies. In the present work the optimal scheduling developed tools results are used to discuss the relationships among the fixed and decision variables and the total operation cost.

For the present study, the considered input variables are:

- total wind resources $(\mathrm{kW})$

- total sun resources $(\mathrm{kW})$

- total forecasted load $(\mathrm{kW})$

- forecasted market price or supply price (m.u./kWh).

The aim of the study is to provide decision makers with relevant information about the way in which their decisions concerning decision variables influence the final operation cost.

Let us consider a decision time horizon of a few hours assuming high precision forecasted values for wind and sun resources, and for the demand. Depending on the context, the market electricity price may be forecasted with lower or higher precision (Zhang, 2005). It is important to note that, among the considered input variables, electricity market price is the most difficult to forecast. However, for a few hours in advance, the market price is already known as the market for day $\mathrm{N}$ is cleared in day $\mathrm{N}-1$.

Alternatively, it may be necessary to consider retailer's supply price if electrical energy is not bought in the pool but supplied by a retailer. In this case, although the contracted tariffs may be known, real time pricing introduces uncertainty in the electricity price. In this relatively short decision time horizon, demand response can be used, if considered interesting, to maintain the operation cost within the aimed limits.

The goals of this study led us to undertake the following analysis of operation cost:

1- Considering fixed wind and sun resources, and electricity price and variable load; 
2- Considering variable electricity price and fixed wind and sun resources, and load.

The operation cost is considered as:

- total operation costs (m.u.)

- LMP (m.u./kWh).

The undertaken studies allow decision makers to determine when demand response should be used to keep operation costs within reasonable limits. Moreover, demand response parameterization can be obtained from the study results when a maximum operation cost (or LMP) is assumed.

Although the proposed methodology can be very useful for aggregators' decision support, it requires relevant computational means. This makes it only suitable for large aggregators. In order to provide medium and small size aggregators with a decision support tool, an ANN based approach is proposed. Section 2.1 presents the reference methodology as a mathematical formulation of the schedule problem, while the ANN based approach is presented in section 2.2.

\subsection{Mixed-Integer Non-Linear Approach}

This sub-section presents the mathematical formulation used for the reference optimization, based on a mixed-integer nonlinear approach implemented in GAMS (GAMS, 2007).

The objective function (1) of the mixed-integer non-linear model is formulated with the aim of finding the total minimal cost of supplying the demand. Equations (2) to (14) refer to the constraints that are considered.

$$
\begin{aligned}
& \text { Minimize } f= \\
& \operatorname{Min}\left(\begin{array}{l}
P_{\text {Main }} \times c_{\text {Main }}+P_{\text {Wind }} \times c_{\text {Wind }} \\
+P_{\text {Photovoltaic }} \times c_{\text {Photovoltaic }}+P_{\text {Other }} \times c_{\text {Other }} \\
-P_{\text {StorageCharge }} \times c_{\text {StorageCharge }} \\
+P_{\text {StorageDischarge }} \times c_{\text {StorageDischarge }} \\
+U_{\text {ndeliveredEnergy }} \times c_{\text {UndeliveredEnergy }} \\
-E_{\text {xcessGeneratedEnergy }} \times c_{\text {ExcessGeneratedEnergy }} \\
+P_{\text {LoadRed }} \times c_{\text {LoadRed }}+P_{\text {LoadRCut }} \times c_{\text {LoadCut }}
\end{array}\right)
\end{aligned}
$$

The equation (2) refers to the first Kirchhoff Law or power balance constraint.

$$
\begin{aligned}
& P_{\text {Main }}+P_{\text {Wind }}+P_{\text {Photovoltaic }}+P_{\text {Other }}+P_{\text {StorageDischarge }} \\
& +U_{\text {ndeliveredEnergy }}+P_{\text {LoadRed }}+P_{\text {LoadCut }} \\
& =P_{\text {Load }}+P_{\text {StorageCharge }}+E_{\text {xcessGeneratedEnergy }}
\end{aligned}
$$

Equations (3) to (8) represent the constraints concerning the maximum capacity considering the available resources, for both generation $(3,5,8)$ and for load response $(6,7)$. Equation (4) imposes that all available photovoltaic energy has to be consumed. Equation (7) represents the participation of loads that can be totally curtailed but not reduced, whereas (6) concerns loads for which the consumption can be reduced.

$$
\begin{aligned}
& P_{\text {Wind }} \leq P_{\text {WindLimit }} \\
& P_{\text {Photovoltaic }}=P_{\text {PhotovoltaicLimit }} \\
& P_{\text {Other }} \leq P_{\text {OtherLimit }} \\
& P_{\text {LoadRed }} \leq P_{\text {LoadRedLimit }} \\
& P_{\text {Cut }}=P_{\text {CutLimit }} \times X_{\text {Cut }} ; X_{\text {Cut }} \in\{0,1\} \\
& P_{\text {Storage }} \leq P_{\text {StorageLimit }}
\end{aligned}
$$

Storage resources require a special treatment due to specific operation constraints. The discharge capacity is considered in equation (9) and the charge capacity in equation (10). In each instant, the battery only can be charging or discharging, as imposed in equation (11).

$$
\begin{aligned}
& P_{\text {StorageDischarge }} \leq P_{\text {StorageDischargeLimit }} \times X_{\text {Storage }} ; X_{\text {Storage }} \in\{0,1\} \\
& P_{\text {StorageCharge }} \leq P_{\text {StorageChargeLimit }} \times Y_{\text {Storage }} ; Y_{\text {Storage }} \in\{0,1\} \\
& X_{\text {Storage }}+Y_{\text {Storage }} \leq 1 ; X_{\text {Storage }} \text { and } Y_{\text {Storage }} \in\{0,1\}
\end{aligned}
$$

It is also necessary to impose that it is not possible to discharge more than the stored energy (12). Similarly, the power to be charged plus the power stored cannot be higher than the total storage resource capacity (13). Finally, the storage state is obtained considering the initial stored energy, the charge, and the discharge in each time period (14).

$$
\begin{aligned}
& P_{\text {StorageDischarge }}-P_{\text {Storage }} \leq 0 \\
& P_{\text {StorageCharge }}+P_{\text {Storage }} \leq P_{\text {StorageLimit }} \\
& P_{\text {Storage }}=P_{\text {Storagelnitial }}-P_{\text {StorageDischarge }}+P_{\text {StorageCharge }}
\end{aligned}
$$

\subsection{ANN-Based Approach}

The use of an Artificial Neural Network (ANN) based approach is proposed with the aim of providing small and medium size players with a decision-support tool that requires modest computational means. The solutions obtained with the methodology presented in section 2.1 are used to train this ANN whose outputs are the total operation cost and the LMP value.

ANN are known as a powerful method for handling nonlinear complex phenomenon, and are able to develop a forecasting model automatically only by training with stored actual data (Hodzic, 2006). Due to this capability, the authors implemented an ANN in Matlab software (MathWorks, 2010) that considers as inputs, the elements in (15).

$\left\{P_{\text {Wind }}, P_{\text {Photovoltaic }}, P_{\text {Load }}, c_{\text {Main }}\right\}$

Therefore, having the forecasted values for wind and solar radiation, and for the load and knowing the electricity price, it is possible to obtain the operation cost (Objective Function - O.F. of the methodology presented in 2.1) and the LMP value as ANN outputs.

Fig. 1 shows the schematic representation of the proposed methodology, which uses Mixed-Integer Non-Linear 
Programming (MINLP) to obtain the optimal scheduling for each scenario. After obtaining a set of scenarios results, the train of the ANN is performed. Finally, the trained network receives as input the variables presented in (15) and devolves the values of operation cost and of the energy component of LMP.

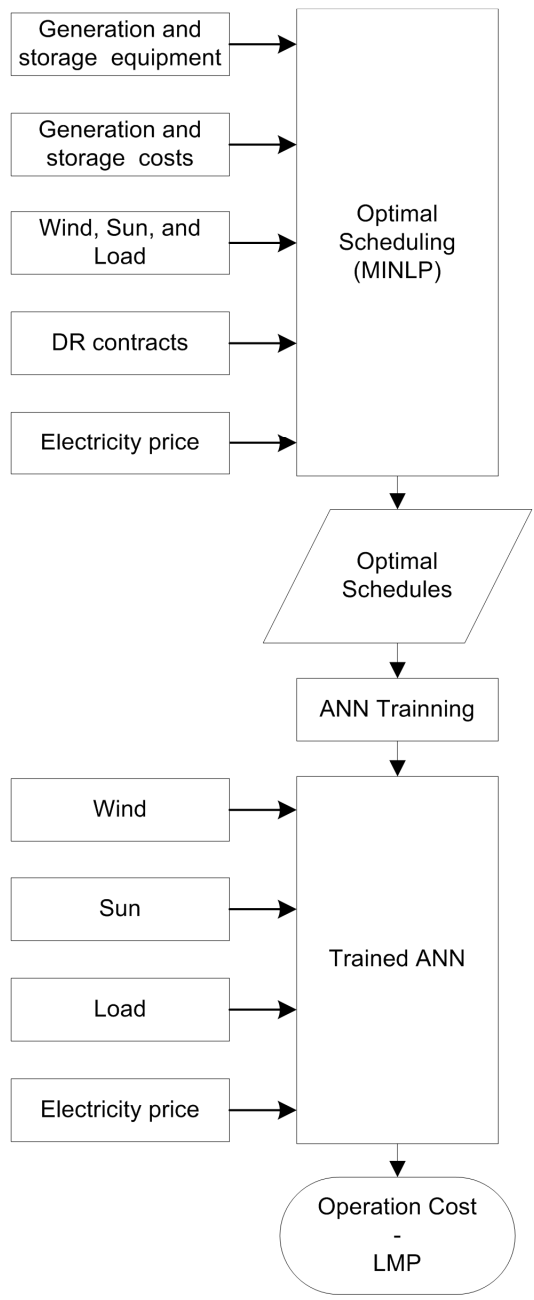

Fig. 1. Block diagram for the proposed methodology.

The structure of the neural network, for both operation cost and LMP values determination, is 4-2-1, i.e. 4, 2, and 1 neurons respectively in input, hidden, and output layers. The used ANN implementation uses Levenberg-Marquardt backpropagation function, updating weight and bias values according to Levenberg-Marquardt optimization. This approach leads to good results, in a good computational time, although requiring more memory than other algorithms (Kecman, 2001). After the training, feed-forward backpropagation algorithm is applied to obtain the forecast.

The obtained results errors can be evaluated using the Mean Absolute Percentage Error (MAPE) that is presented in equation (16).

MAPE $=\frac{1}{n r} \sum_{r=1}^{n r}\left(\frac{\left|R_{r}^{\text {reference }}-R_{r}^{\text {proposed }}\right|}{R_{r}^{\text {reference }}}\right) \times 100$

The index 'reference' is related with the reference optimization solution (obtained using MINLP). The index 'proposed' is related with the results of the ANN approach. Smaller values of MAPE mean that the solutions obtained with the ANN approach are closer to the solutions obtained with the reference optimization method.

\section{CASE-STUDY}

Let us consider two scenarios - Case A and Case B - for the illustration of the proposed methodology. The proposed ANN based methodology is used to determine the value of the objective function (O.F.) and the energy component of the Locational Marginal Price (LMP). The O.F. value corresponds to the total cost of supplying the forecasted load. The validation of the results is done by comparing the results obtained using the proposed methodology with the results obtained with the Mixed-Integer Non-Linear Programming (MINLP) approach.

\subsection{Data characterization}

The input data include load, wind, photovoltaic, and energy prices values. The load values are obtained from historical consumption data of the Portuguese distribution company (EDP). This includes the consumption of 229 medium voltage customers, which was collected in a period of 3 months in summer and 3 months in winter for working days and weekends (Ramos et al., 2007).

The ANN training has been done using a data set characterized in Table 1. Several training arrays resulting from the variation the load, the wind and the photovoltaic power, and the energy price, between a minimum and a maximum value have been considered, as presented in Table 1. The column including the step value indicates how each variable is diversified to obtain different train arrays corresponding to different system conditions.

Table 1. ANN training set

\begin{tabular}{cccc}
\hline \hline & Minimum & Maximum & Step \\
\hline Load $(\mathrm{kW})$ & 10000 & 60000 & 1000 \\
\hline Wind $(\mathrm{kW})$ & 0 & 20000 & 2000 \\
\hline PV $(\mathrm{kW})$ & 0 & 1500 & 250 \\
\hline Price $($ m.u. $/ \mathrm{kWh})$ & 0.04 & 0.15 & 0.01 \\
\hline \hline
\end{tabular}

Table 2 presents the two cases to be analyzed. In Case A, load, wind, and photovoltaic are fixed and the energy price varies. For Case B, the variation is in the load and the other variables are fixed. The number of the output variable values to be determined in each case is 12 and 40, respectively for Case A and Case B.

Table 2. Data set for Case A and Case B

\begin{tabular}{ccc}
\hline \hline & Case A & Case B \\
\hline Load $(\mathrm{kW})$ & 45000 & $15000-55000$ \\
\hline Wind $(\mathrm{kW})$ & 10000 & 14000 \\
\hline $\mathrm{PV}(\mathrm{kW})$ & 1000 & 1500 \\
\hline Price $($ m.u. $/ \mathrm{kWh})$ & $0.04-0.15$ & 0.1 \\
\hline \hline
\end{tabular}

This case-study, concerning the formulation presented in the previous section, considers the following fixed costs, in 
monetary units (m.u.): 0.045, 0.2, 0.055, 0, 0.08, 3, 0.2, 0.09 , and 1.2, respectively for wind, photovoltaic, other generation, storage charge, storage discharge, undelivered energy, excess generated energy, load reduction, and load curtailment.

\subsection{Results}

The results of the case-study are presented in Fig. 2 (for Case $A$ ) and in Fig. 3 (for Case B). An evaluation of the results is made comparing the execution time and the Mean Absolute Percentage Error (MAPE) between the two methods (MINLP and ANN) for both cases A and B. MINLP is therefore used as the reference method to validate ANN results. This evaluation is presented in Table 3 and Table 4.

ANN had an excellent result for lower energy prices, but when the energy price became higher and LMP stabilizes in a fixed value, the results became lower than the reference values. In these conditions, one can conclude that when the load forecast is fixed and results are obtained in function of energy price variation, the results of LMP for higher energy prices are worse.

For Case B, the results were obtained in function of a variation of load between $15 \mathrm{MW}$ and $55 \mathrm{MW}$. The other variables were fixed as shown in Table 2. The O.F. results were, as can be seen in Fig. 3, close to the reference values, except for higher values of load for which ANN values are lower than the reference values. In what concerns the LMP, for lower values of load and therefore for LMP lower values, the results are lower than the reference values but close to them. When we look to higher values of load, the LMP values obtained are very different from the reference values, and always above those values. This can be due to the fact that, for those load values, LMP values increase significantly, with a little variation of load. As this is a very specific region of the training set, it is not sufficiently trained. These problems could be solved implementing a different neural network, dedicated to a specific range of load values.

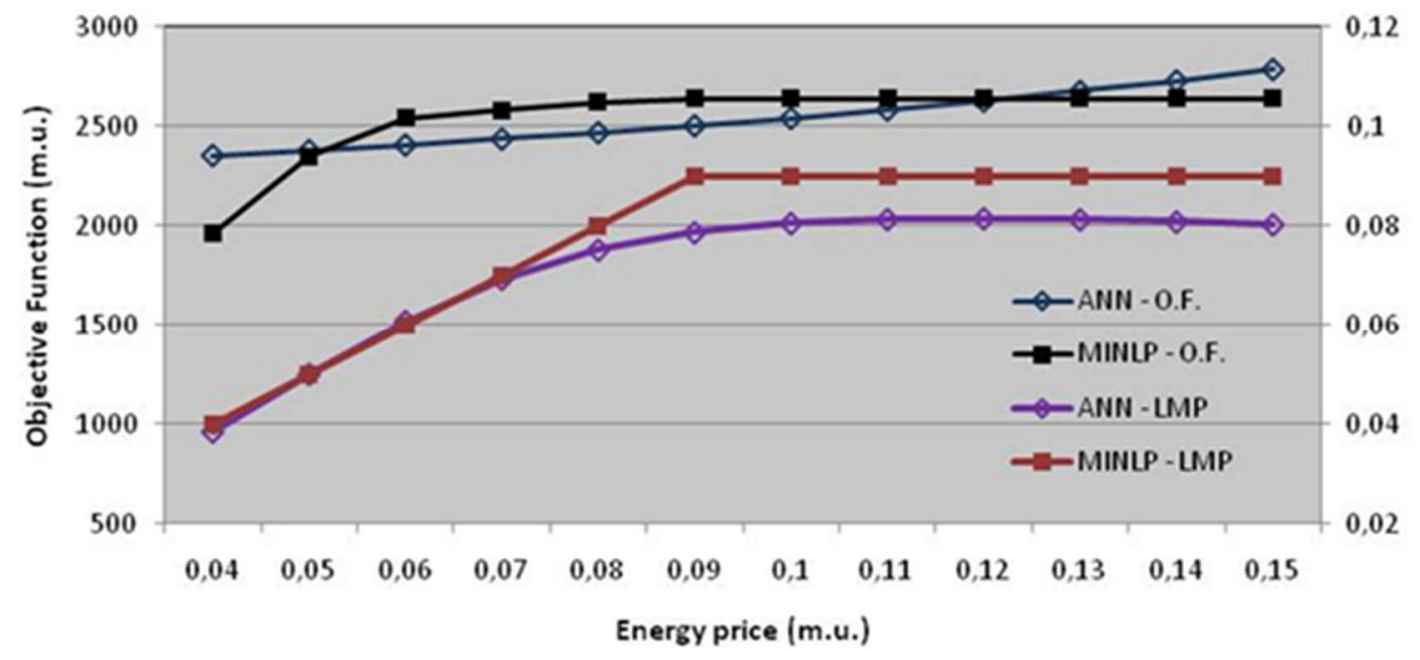

Fig. 2. Results for Case A.

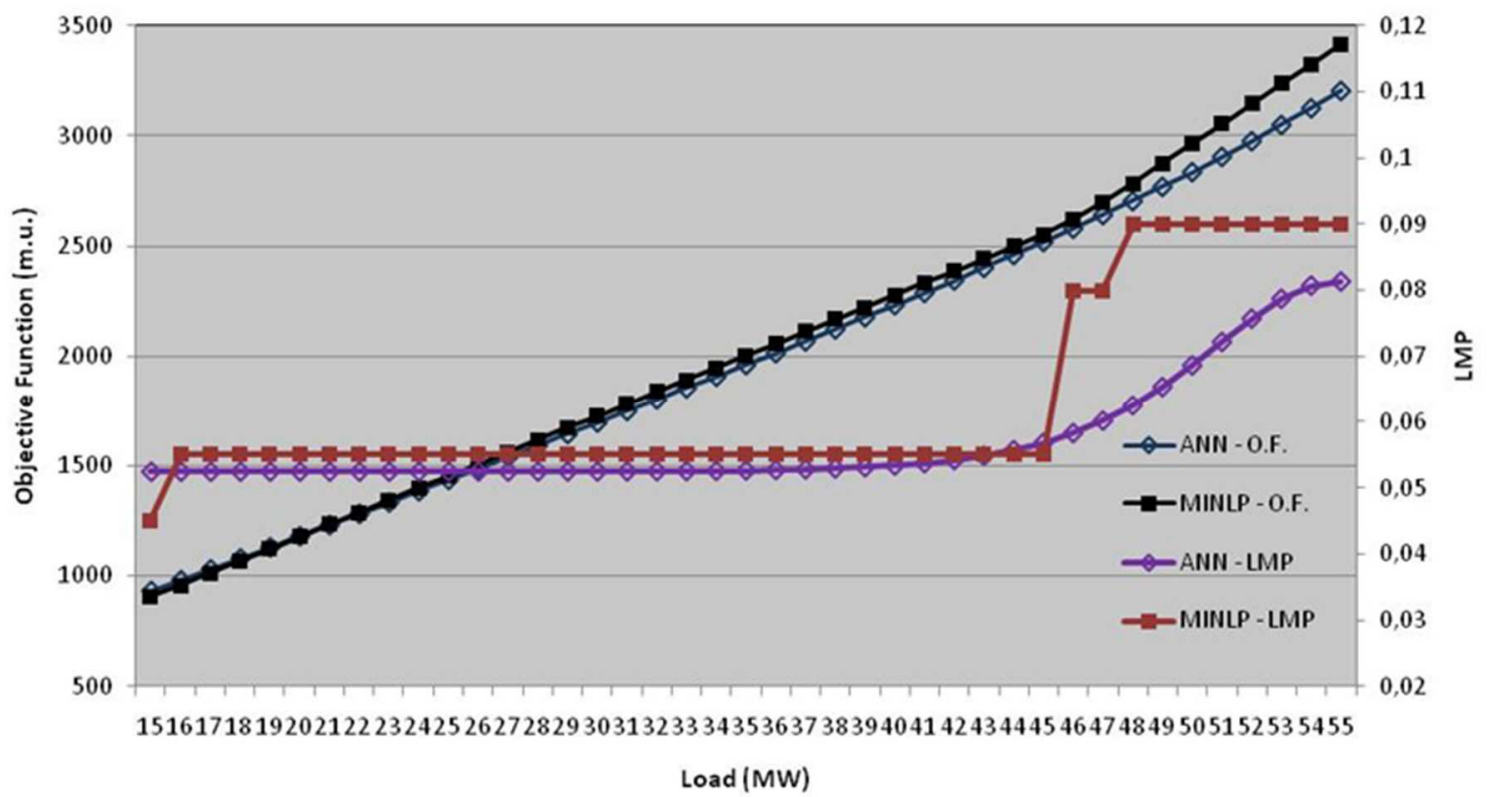

Fig. 3. Results for Case B. 
Analyzing the values of Table 3 that presents the execution times, it is easy to conclude that the ANN based approach gives results much faster than MINLP. Moreover, while MINLP processing time depends on the dimension of the problem, ANN can give results for different problem dimensions practically in the same execution time. Obviously, these results do not contemplate the execution time of training the neural network.

Table 3. Execution times in seconds

\begin{tabular}{ccc}
\hline \hline & Case A & Case B \\
\hline MINLP & 1.774 & 5.557 \\
\hline ANN & 0.032 & 0.032 \\
\hline
\end{tabular}

Using the results of the MINLP approach as the reference values, MAPE has been calculated using (16). MAPE values are presented in Table 4 . In both cases $A$ and $B$, as discussed above, the better results were obtained for the O.F. However, in spite of the worse MAPE values, the results obtained for LMP are also satisfactory for lower values of load and energy price. Being a mean value, MAPE introduces some ambiguity in results evaluation.

Table 4. MAPE results (\%)

\begin{tabular}{ccc}
\hline \hline & Case A & Case B \\
\hline O.F. & 4.986 & 2.216 \\
\hline LMP & 7.099 & 8.246 \\
\hline \hline
\end{tabular}

\section{CONCLUSIONS}

Future power systems, operating in the context of intensive use of DER and in a competitive market environment, require optimized resources management. This is relatively easy to larger players, with financial means to have access to adequate decision support tools to support decision making concerning their optimal resource schedule. However, the smaller players have difficulties in accessing this kind of tools. So, it is required that these smaller players can be offered alternative methods to support their decisions.

The present paper has proposed an ANN based methodology to determine the value of the objective function that represents the cost of supplying the required load in a power system, and the energy component of the Locational Marginal Price. This methodology allows obtaining the required results with modest computational means. A reference optimization methodology (mixed-integer nonlinear programming) was used to obtain the ANN training set and to compare the results obtained with the proposed method. Results show that the ANN approach can provide good results for cost calculation. Moreover, execution time of this approach is much lower than that of the reference method. In what concerns the LMP calculation, ANN can give excellent results in a specific range of input values. Further work will focus on using different neural networks in specific ranges of input data the LMP calculation.

\section{ACKNOWLEDGEMENT}

The authors would like to acknowledge FCT, FEDER, POCTI, POSI, POCI, POSC, POTDC and COMPETE for their support to R\&D Projects and GECAD Unit.

\section{REFERENCES}

GAMS Development Corporation (2007), Washington, DC 2007, USA. GAMS - the solver manuals.

Hodzic, N., Konjic, T., Miranda, V. (2006), Artificial Neural Networks Applied To Short Term Load Diagram Prediction, 8th Seminar on Neural Network Applications in Electrical Engineering 2006, pp.219-223, 25-27 September 2006.

Kecman, V., "Learning and Soft Computing: Support Vector Machines, Neural Networks, and Fuzzy Logic Models", A Bradford book, 2001, ISBN 0-262-11255-8, 2001.

Liu, X., Su, B. (2008), Microgrids - an integration of renewable energy technologies, China International Conference on Electricity Distribution, 2008. (CICED 2008), pp.1-7, 10-13 December, 2008.

MathWorks (2010), Matlab Software, available online from: http://www.mathworks.com/, visited in September, $20^{\text {th }}$, 2010 .

Milošević, M., Andersson, G. (2005), Generation Control in Small Isolated Power Systems, 37th Annual North American Power Symposium, 2005.

Morais, H., Kadar, P., Cardoso, M., Vale, Z. A., Khodr, H.M. (2008), VPP operating in the isolated grid, IEEE Power and Energy Society General Meeting 2008 , pp.1-6, 2024 July 2008.

Morais, H., Kadar, P., Faria, P., Vale, Z. A., Khodr, H. M. (2010), Optimal scheduling of a renewable micro-grid in an isolated load area using mixed-integer linear programming, Renewable Energy, Vol.35, No. 1, pp151156, January 2010.

Ramos, S., Vale Z., Santana J. And Duarte J. (2007), Data Mining Contributions to Characterize MV Consumers and to Improve the Suppliers-Consumers Settlements. PES GM 07 - IEEE Power Engineering Society, Tampa, Florida, USA, 24-28 July.

REN21 (2010), Renewable Energy policy Network for the $21^{\text {st }}$ Century, Global Trends in Green Energy 2009, available online from: http://www.ren21.net/globalstatusreport/REN21_GSR_2 010_Press_Release.pdf , visited in September, $20^{\text {th }}$, 2010.

Sioshansi, R., Short, W. (2009), Evaluating the Impacts of Real-Time Pricing on the Usage of Wind Generation, IEEE Transactions on Power Systems, Volume 24, Issue 2, pp. 516-524, May 2009.

Vale, Z., Ramos, C., Morais, H., Faria, P., Silva, M. (2009), The role of demand response in future power systems, IEEE - T\&D Asia 2009, Seoul, Korea, 27 - 30 October 2009.

Zhang, L., Zhao, J., Han, X., Niu, L. (2005), Day-ahead Generation Scheduling with Demand Response, IEEE/PES Transmission and Distribution Conference and Exhibition: Asia and Pacific, pp.1-4, 2005. 